\title{
Supercomputer analysis of social, epidemiological and economic processes
}

\author{
O. Krivorotko \\ Institute of Computational Mathematics and Mathematical Geophysics SB RAS, Novosibirsk, Russia \\ Novosibirsk State University, Novosibirsk, Russia \\ *e-mail: krivorotko.olya@mail.ru
}
Key words: inverse problems, mathematical modelling, social processes, epidemiology, economy, optimization, Hamilton-Jacobi-Bellman equation, Fokker-Plank equation, dynamic programming

Motivation and Aim: The specifics of the dissemination of information in society, the development of socially significant diseases (tuberculosis, HIV / AIDS) and economic processes depend on the region. One of the most effective methods is the development and identification of mathematical models that describe the processes of information dissemination in social networks [1], infections in the population [2] and economic processes [3]. Such models are described by systems of differential equations, the coefficients of that characterize the distribution of information, population, disease development and economic processes in the country. To control information in social networks, epidemics in individual regions and economic processes, it is necessary to refine the model coefficients by some additional information (the inverse problem) [4]. Methods and Algorithms: One way to solve the problem of improving the coefficients is to reduce the inverse problem to a variational formulation, where the functional satisfies to the Hamilton-Jacobi-Bellman partial differential equation, or characterizes the quadratic deviation of the model data from the experimental ones for systems of stochastic differential equations or partial differential equations. The Tikhonov regularization, gradient methods and stochastic approach (genetic algorithm, simulated annealing) are used for solving ill-posed inverse problems. To reconstruct the control function the Pontryagin maximum principle and dynamic programming are used.

Results: The reconstructed coefficients and control functions in mathematical models of social, epidemiological and economic processes allows one to refine the prognosis of dynamic of processes and give the recommendation for control its.

Conclusion: A prototype of the digital Earth globe will be created with the possibility to visualize social, epidemiological and economic processes in various countries and continents based on mathematical modeling dynamic plug-in.

Acknowledgements: Supported by the Ministry of Education and Science of Russian Federation, by the President Grant of Russian Federation (No. MK-1214.2017.1) and by the grant No. 18-71-10044 of Russian Scientific Found (RScF).

\section{References}

1. Dai G., Ma R., Wang H., Wang F., Xu K. (2015) Partial differential equations with robin boundary condition in online social networks. Discrete and Continuous Dynamical Systems Series B. 20(6):16091624.

2. Kabanikhin S.I., Krivorotko O.I., Kashtanova V.N. (2018) A combined numerical algorithm for reconstructing the mathematical model for tuberculosis transmission with control programs. Journal of Inverse and Ill-Posed Problems. 26(1):121-131.

3. Herowitz J. (2014) Ill-posed inverse problems in economics. Annual Review of Economics. 6:21-51.

4. Kabanikhin S.I. (2008) Definitions and examples of inverse and ill-posed problem. Journal of Inverse and Ill-Posed Problems. 16(4):317-357. 